\title{
ATENÇÃO EM SAÚDE BUCAL NA CRECHE SORENA: RELATO DE EXPERIÊNCIA DE 17 ANOS PROMOVENDO SAÚDE EM PRÉ-ESCOLARES
}

\section{ORAL HEALTH CARE AT SORENA NURSERY: AN EXPERIENCE REPORT OF 17 YEARS PROMOTING HEALTH IN PRESCHOOL}

\section{RESUMO:}

O objetivo deste artigo é relatar a experiência de 17 anos de atividades de promoção de saúde bucal realizadas com pré-escolares inseridos no Projeto de Extensão "Atenção Integral em Saúde Bucal na Creche Sorena", desenvolvido a partir de 2001. O Projeto foi aprovado pelo Parecer de Etica 044/2007 'FO-UFPA. As atividades executadas por 3 cirurgiãs dentistas odontopediatras, 1 aluna bolsista, 12 alunos voluntários e 3 docentes da Faculdade de Odontologia da Universidade Federal do Pará visam promover o aprendizado em saúde bucal e proporcionar a prevenção e recuperação da saúde nos préescolares de 2 a 5 anos por meio de ações de promoção, prevenção e recuperacão de saúde bucal. Atividades lúdicas como jogos, fantoches, contacão de estórias e palestras foram realizadas para promover a construcão de conhecimentos. $\mathrm{O}$ projeto, no decorrer de 17 anos, obteve resultados positivos. As experiências vivenciadas por seus integrantes, a percepção dos pré-escolares, seus pais e/ou responsáveis, professores e funcionarios da creche foram relatadas de maneira reflexiva, demonstrando a importância de manter atividades de educação em saúde. Os trabalhos desenvolvidos para acolhimento dos préescolares refletiram em um comportamento mais tranquilo e menos traumático, perceptível visualmente durante os atendimentos odontológicos.

Palavras-chave: Pré-Escolar; Estudantes de Odontologia; Educação em Saúde; Promoção da Saúde; Saúde Bucal.

\begin{abstract}
:
This paper aims to report the experience of 17 years of the extension project "Integral Attention to Oral Health in Sorena Day Care Center" which provides activities to improve oral health of preschool children since 2001. The project was approved by the Research Ethics Committee 044/2007 FO-UFPA. The activities have being developed by 3 pediatric dentists, 1 undergraduate scholarship holder, 12 undergraduate students, through volunteer work, and 3 professors, members of the School of Dentistry from Federal University. of Pará. The activities aim to provide learning about oral health as well as health prevention and treatment to preschoolers from 2 to 5 years of age through actions of oral health promotion, prevention and treatment. It were carried out through recreational activities such as games, puppets, storytelling and lectures in order to construct knowledge. It is possible to conclude that during 17 years the project has being achieving positive results. The experiences of the ones that take part in the project, the perception of the preschool children, their parents and/or guardians, teachers and day care staff were reported in a reflexive manner, demonstrating the importance of creating new possibilities to health education. It was possible to note that the activities developed to welcome preschoolers kept them calmer and as a result the dental procedures were less traumatic to them.
\end{abstract}

Keywords: Preschool Children; Dental Students; Health Education; Health Promotion; Oral Health.

${ }^{* *}$ Professora da Universidade Federal do Pará (UFPA), Belém - PA, Brasil. E-mail: dtemmi@ufpa.br

${ }^{* * *}$ Professor da Universidade Federal do Pará (UFPA), Belém - PA, Brasil. E-mail: helder@ufpa.br

${ }^{* * * *}$ Professora da Universidade Federal do Pará (UFPA), Belém - PA, Brasil. E-mail: rebar@ufpa.br

${ }^{* * * * * *}$ Professor da Universidade Federal do Pará (UFPA), Belém - PA, Brasil. E-mail: izamir@ufpa.br

${ }^{* * * * * *}$ Professora da Universidade Federal do Pará (UFPA), Belém - PA, Brasil. E-mail: marizeli@ufpa.br
} 


\section{Introdução}

A promoção de saúde supõe uma concepção que não restrinja a saúde apenas à ausência de doença, mas que seja capaz de atuar sobre seus determinantes, atingindo as condições de vida da população, a prestação de serviços clínico-assistenciais, considerando as ações intersetoriais que envolvam a educação, o saneamento básico, a moradia, a renda, o trabalho, a alimentação, o meio ambiente, o acesso a bens e serviços essenciais, assim como o lazer, entre outros determinantes sociais da saúde (SÍCOLI; NASCIMENTO, 2003).

Uma das concepções de promoção da saúde, que entende a saúde como ausência de doença, ressalta a responsabilidade dos indivíduos em preservar a sua saúde por meio de hábitos saudáveis, sem que seja posta em discussão a real possibilidade da adoção de tais hábitos (IGLESIAS; ARAUJO, 2011).

A partir da década de 1980, a promoção de saúde passou a ganhar destaque no campo da Saúde Pública, tendo o conceito sido introduzido oficialmente pela Organização Mundial de Saúde - OMS (WHO, 1984).

A Carta de Ottawa foi um marco importante, inspirada pelos princípios da Declaração de Alma Ata (1978) e pela meta "Saúde para todos no ano 2000". Dentre os campos de ação da promoção de saúde mencionados na Carta de Ottawa estão o estabelecimento de políticas públicas favoráveis à saúde, a criação de ambientes propícios, o fortalecimento da ação comunitária, o desenvolvimento de habilidades pessoais e a reorientação dos serviços sanitários (BRASIL, 2002).

Como afirmado na Carta de Ottawa, a promoção tem como objetivo garantir o acesso universal à saúde e está de acordo com o princípio de justiça social. "Alcançar a equidade consiste em eliminar as diferenças desnecessárias, evitáveis e injustas que restringem as oportunidades para se atingir o direito de bem-estar" (BRASIL, 2002).

A promoção da saúde relaciona-se ao alívio do sofrimento das pessoas, e essa relação, apesar de não estar clara em sua definição, é muito importante para os profissionais da saúde. Em busca do alívio do sofrimento, existem ações que pertencem ao campo dos serviços de saúde e ações externas aos serviços de saúde. Assim, a promoção da saúde para os serviços de saúde inclui as ações preventivas ou assistenciais envolvidas no ato de cuidar. Dessa forma, ao relacionar a promoção da saúde ao alívio do sofrimento, seria possível construir estratégias mais efetivas para os serviços de saúde (NORMAN, 2013).

A saúde bucal é influenciada por diversos fatores, como: condições sociais e econômicas da população, pequena parcela de investimento que a área recebe e a falta de informação sobre os cuidados básicos de saúde (MURRAY, 2005).

Segundo Moysés e Watt (2000), a promoção de saúde bucal refere-se, fundamentalmente, às ações destinadas às necessidades da população. Atribui-se ao profissional da área odontológica a obrigatoriedade de implantar estratégias de promoção de saúde, onde a educação em saúde bucal é uma dessas estratégias. Assim, a educação em saúde bucal está relacionada às experiências de aprendizagem, fortalecidas pelo conhecimento, conscientização e produção de habilidades necessárias para obtenção da saúde bucal.
A promoção de saúde bucal pode ser desenvolvida em uma enorme diversidade de espaços sociais, grupos populacionais e atividades, por diferentes profissionais. Nas escolas, por exemplo, pode-se desenvolver uma série de ações para promover saúde bucal, como, por exemplo, uma política de alimentação, oferecendo alimentos saudáveis na cantina; a inclusão de tópicos de saúde bucal no currículo, destacando informações práticas, não só a discussão sobre a importância de limpeza dos dentes, mas também como limpar, associadas à disponibilidade de espaços adequados para a higienização dos dentes (MESQUINI et al., 2006).

$\mathrm{O}$ treinamento de professores sobre tópicos de saúde bucal contribui para promover a saúde bucal em escolas. Isso permite a aproximação da escola e comunidade, favorecendo a ampliação das relações com os pais e o desenvolvimento de projetos de saúde (MESQUINI et al., 2006).

A escola passa a ser um ambiente de suporte para a saúde, através de um trabalho coletivo e participativo. As práticas de saúde desenvolvidas nos primeiros cinco anos de vida irão determinar o padrão de saúde bucal durante a vida futura. Bons hábitos estabelecerão uma boa condição de saúde bucal, enquanto os maus hábitos praticados nesta fase dificilmente se transformarão no futuro. A implantação de uma política universal e com serviços integrais de saúde bucal para inclusão de crianças na primeira infância é prejudicado pelo atendimento odontológico, que, geralmente, é baseado em ações intervencionistas e curativas (FREIRE, 2000).

Levantamentos epidemiológicos que incluam a faixa etária de 18 a 36 meses e a idade índice de 5 anos são importantes para verificar as condições de saúde bucal da dentição decídua. O Projeto SB Brasil 2003 observou que aproximadamente $27 \%$ das crianças de 18 a 36 meses apresentavam pelo menos um dente decíduo com experiência de cárie dentária, situação que se propagava a quase $60 \%$ das crianças aos 5 anos de idade, indicando um ceo-d igual a 1,07 aos 18 a 36 meses e de 2,80 aos 5 anos (BRASIL, 2004).

Para a construção do Projeto de Extensão "Atenção Integral em Saúde Bucal na Creche Sorena", levou-se em consideração o projeto pedagógico da Faculdade de Odontologia do Instituto de Ciências da Saúde da Universidade Federal do Pará (UFPA), que tem como objetivo graduar um cirurgião-dentista generalista com sólida formação técnica, científica, humanística e ética, orientado para a promoção de saúde com ênfase na prevenção de doenças bucais prevalentes e consciente da necessidade de educação continuada, interagindo com a população, capaz de alterar o perfil epidemiológico de saúde bucal da região, participando do sistema de saúde, com capacidade de liderança e sensibilidade social (UFPA, 2000).

Assim, o objetivo desse artigo é relatar a experiência de 17 anos das atividades de promoção de saúde bucal em pré-escolares, refletindo sobre a importância dessas ações para a melhora da condição de saúde bucal e aceitação ao tratamento odontológico, verificada durante o desenvolvimento do Projeto de Extensão "Atenção Integral em Saúde Bucal na Creche Sorena", executado pela Faculdade de Odontologia da Universidade Federal do Pará. 


\section{Material e Métodos}

O Centro Sócio Recreativo de Nazaré/Creche Sorena, fundado em 29 de junho de 1970, integra as Obras Sociais da Basílica Santuário de Nazaré, atualmente coordenada pelas Missionárias de Santa Terezinha. A entidade possui perfil de sociedade civil, sem fins lucrativos de caráter beneficente, educativo, cultural e de assistência social, com finalidade de proporcionar o bem-estar e desenvolvimento integral de 120 crianças de 2 a 5 anos de idade por ano, em média. A Creche funciona em regime de tempo integral. Em parceria com Universidade Federal do Pará, o atendimento odontológico tornou-se uma das atividades desenvolvidas na Creche por meio do Projeto de Extensão (CORRÊA, 2016). O Projeto foi aprovado pelo Parecer 044/2007 da Comissão de Ética da Faculdade de Odontologia da Universidade Federal do Pará.

As atividades executadas pelo Projeto ocorrem nas salas de aula, salão de convivência, bancada de higiene bucal e no consultório odontológico da Creche, durante o turno da manhã, para não interferir na rotina da instituição, com a participação atual de 3 cirurgiãs dentistas odontopediatras, 1 aluna bolsista, 12 alunos voluntários e 3 docentes da Faculdade de Odontologia da UFPA (FO - UFPA).

O Projeto de Extensão, desde a sua implantação, em 2001 (Figura 1), promove a saúde bucal em préescolares através da realização de atividades educativas, preventivas e curativas, proporcionando uma atenção odontológica integral, com controle da incidência da cárie dentária e doença periodontal. Necessidades de tratamento especializadas são referenciadas para a FO-UFPA. Para a demanda apresentada, organiza-se um cronograma com atividades odontológicas, semanalmente.

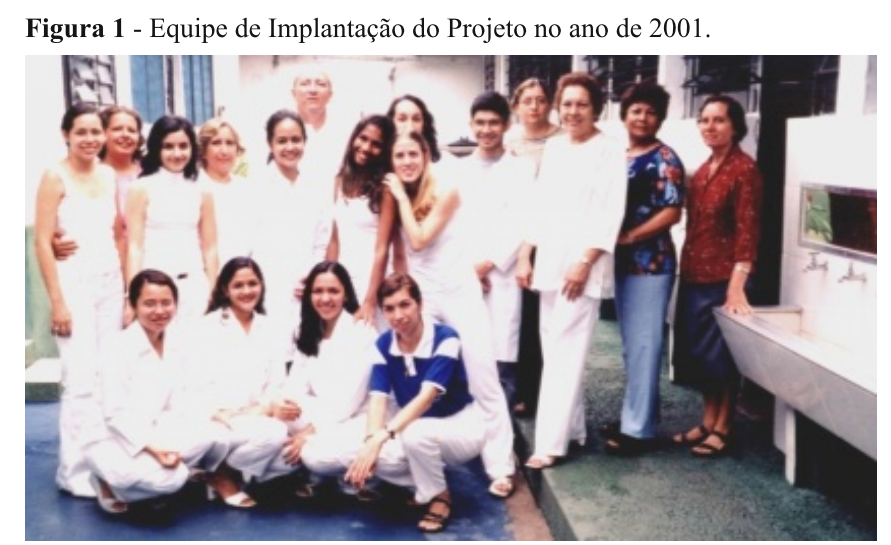

Fonte: Autores.

As ações em saúde bucal desenvolvem-se a partir de observações feitas sob a rotina escolar, observando os horários das refeições, o tipo de alimentação e os hábitos de higiene bucal, já que as crianças ficam na creche no horário de 8 às 16 horas. No período de permanência, as crianças fazem três refeições (café, almoço e lanche), elaboradas por nutricionista com orientação da equipe do projeto sobre os alimentos cariogênicos.

Os recursos metodológicos empregados para o desenvolvimento das atividades propostas levam em conta o público-alvo, formado por crianças de 2 a 5 anos e de baixa renda. Por este motivo, atenta-se para fatores importantes, tais como: o tempo de duração das atividades, o uso de linguagem e termos apropriados, com temas de interesse sobre assuntos em pauta e o uso de imagens coloridas e significativas, de forma descontraída e que despertam o interesse e atenção das crianças da Creche (OLIVEIRA, 2014).

Com isso, são desenvolvidos e aperfeiçoados pela equipe odontológica os métodos e recursos materiais utilizados nas atividades realizadas: palestras, escovação supervisionada (Figura 2), oficinas de esclarecimentos e motivação à higiene oral, entrega de escovas e brindes (balões, jogos educativos, entre outros), após as ações realizadas, e produção de instrumentos audiovisuais, como músicas incentivadoras e estórias.



Fonte: Autores.

As atividades lúdicas (Figuras 3 e 4 ) são importantes para a interação e comunicação. São ministradas palestras educativas, tanto para as crianças como para pais ou responsáveis, sobre temas relacionados à importância da saúde bucal, etiologia e prevenção da cárie dentária, dieta cariogênica, alimentos protetores, doença periodontal e a técnica de escovação.

As crianças e as pessoas de seu convívio diário (professores e responsáveis) são motivados para o exercício de hábitos saudáveis, que interfiram no curso das doenças bucais, evitando o estabelecimento da doença e contribuindo para a diminuição da prevalência da doença cárie e periodontal.


Fonte: Autores.

Capacitam-se também as professoras sobre as práticas de saúde bucal, como a higiene bucal, utilizando a técnica de Fones e orientando sobre a higienização da língua e o uso de creme dental fluoretado na concentração padrão de 1.000 a 1.100 ppm, com o devido cuidado em relação à quantidade para evitar o aparecimento de fluorose dentária (OLIVEIRA et al., 2012), assim como a correta utilização do fio dental e os cuidados para substituição das escovas dentais a cada três meses, ou quando as cerdas desfiarem (ADA, 2011). Essas orientações 
também são repassadas para os pais ou responsáveis.

Nas atividades preventivas, além da escovação supervisionada, são utilizados selantes de cicatrículas e fissuras resinosos e substâncias com flúor. Assim, a aplicação tópica de flúor é realizada coletivamente pelos menos duas vezes ao ano. Crianças com alto risco tem aplicações individuais de acordo com a necessidade (BRASIL, 2009).

Ressalta-se que a cidade de Belém não tem água de abastecimento fluoretada desde 2010.

Os procedimentos curativos, como restaurações com resina composta e tratamento restaurador atraumático com cimento de ionômero de vidro e extrações, são realizados de acordo com a necessidade de tratamento de cada criança examinada, proporcionando o estabelecimento e a devolução de uma condição de saúde bucal favorável. Em caso de necessidade de instalação de mantenedor de espaço, a criança é encaminhada para a FO-UFPA (Figura 5).

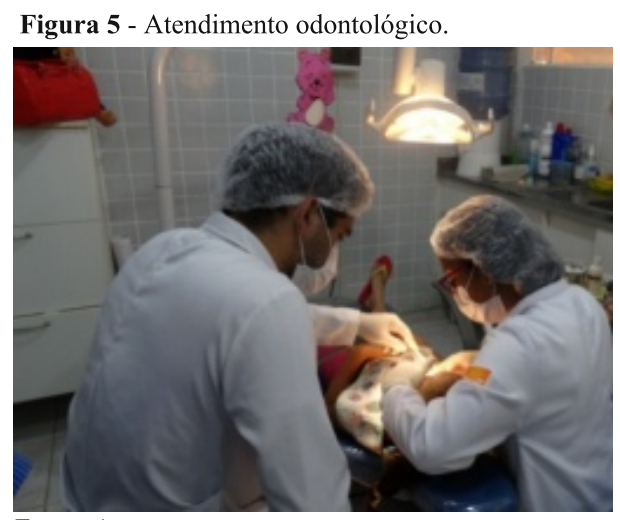

Fonte: Autores.

\section{Resultados e discussão}

No decorrer das atividades realizadas pelo projeto, ocorreu nas crianças o desenvolvimento do hábito de práticas de higiene bucal, com percepção da diminuição do comportamento negativo dos préescolares em relação ao atendimento odontológico. Isso foi relatado também pelos pais e professores, que passaram a colaborar para o alcance e manutenção da saúde bucal de seus filhos e alunos.

As atividades realizadas para educação e promoção de saúde bucal em crianças são de grande relevância, pois favorecem a relação entre os profissionais de odontologia e os escolares (VENÂNCIO et al., 2011), reduzindo a ansiedade e o medo, promovendo, assim, a eficácia da intervenção odontológica de forma leve, descontraída e menos traumática para as crianças (BARRETO et al., 2013).

Atividades lúdicas como peças teatrais, contação de estórias, jogos e brincadeiras realizadas frequentemente com as crianças propiciaram uma maior participação das mesmas nas ações desenvolvidas pelo projeto. A equipe odontológica desenvolveu várias estratégias para melhorar o relacionamento entre os envolvidos no projeto. Assim, foi possível verificar que as atividades lúdicas voltadas para as crianças e as palestras para seus responsáveis promoveram efeitos positivos sobre a saúde bucal das crianças. Isso também pode ser observado por outros estudos (BUENO et al., 2012; SIQUEIRA et al., 2010).
Através das atividades de escovação supervisionada com dentifrício fluoretado, realizadas duas vezes ao dia na creche pela equipe odontológica e professores, e em casa pelos pais ou responsáveis, conseguiu-se criar o hábito de higiene bucal nos pré-escolares e o uso de substâncias com flúor (flúor em gel e verniz fluoretado), observando o risco de cárie do pré-escolar (BRASIL, 2009), o que foi eficaz nos procedimentos preventivos. Ao longo do desenvolvimento do projeto, houve a percepção quanto à melhoria da condição de saúde bucal em relação à diminuição do biofilme, carie dentária e gengivite dos menores atendidos no projeto.

A Odontologia em Saúde Coletiva, exercida com ações de educação e promoção de saúde alicerçada em tecnologias leves, é uma maneira eficaz e econômica de evitar o aparecimento ou agravamento de lesões bucais. Essas ações, pautadas na integralidade da atenção, no cuidado em saúde, tanto nas unidades de saúde como nos espaços comunitários, e na responsabilização da população adstrita, são atribuições comuns a todos os membros de uma equipe de saúde, podendo ser desenvolvidas por qualquer profissional (BRASIL, 2007).

Ressalta-se a incorporação de hábitos de higiene bucal à rotina da creche e o auxílio dos professores como multiplicadores de conhecimento ao longo da atuação do projeto, corroborando com resultados encontrados em outros projetos em que os professores participam das atividades de saúde bucal (SANTOS et al., 2012). A atuação da equipe envolvida no cuidado das crianças foi incentivadora para mudanças relativas às práticas educativas e preventivas, assim como também para aguçar conhecimentos sobre a importância de uma alimentação saudável.

Um programa educacional de promoção da saúde bucal que empregue hábitos saudáveis exigirá menos recursos do que as medidas para modificar posteriormente os hábitos deletérios e efeitos (HAUSEN et al., 2007).

Outro fator importante na realização do Projeto foi a participação dos alunos de Odontologia na execução de todas as atividades propostas no Projeto.

Ao longo dos 17 anos de desenvolvimento do projeto, aproximadamente 200 alunos, 5 bolsistas e 8 professores participaram do projeto, que já gerou cinco trabalhos de conclusão de curso e vários trabalhos apresentados em eventos científicos com publicações em anais. Os alunos envolvidos tiveram a oportunidade de praticar de uma odontologia integral, vivenciando o tripé ensino, pesquisa e extensão, contribuindo para sua formação profissional, fortalecendo o perfil do CD proposto no projeto pedagógico do Curso de Odontologia da UFPA (UFPA, 2000).

\section{Conclusão}

O Projeto, no decorrer de 17 anos, conseguiu obter resultados positivos, perceptíveis na melhora de saúde bucal dos pré-escolares assistidos pela Creche Sorena, demonstrando, assim, a importância de manter as atividades de educação em saúde bucal. Os trabalhos desenvolvidos para o acolhimento dos escolares da Creche mostraram-se eficazes, refletindo nas crianças um comportamento mais leve e menos 
traumático perante os atendimentos odontológicos.

As atividades do projeto também vêm conseguindo promover a capacitação dos professores da Creche, tornando-os parceiros na promoção de saúde bucal. Pais e responsáveis também assumiram sua responsabilidade como promotores e mantenedores de saúde. Os alunos de graduação envolvidos no Projeto tiveram participação em atividades de pesquisa, ensino e extensão, fortalecendo sua formação profissional e futura inserção no mercado de trabalho.

\section{Referências}

AMERICAN DENTAL ASSOCIATION. Statement on Toothbrush Care: cleaning, storage and replacement. Council on Scientific Affairs. 2011. Disponível em: http://www.ada.org/1887.aspx. Acesso em: 10 nov. 2018.

BARRETO, R.A.; CARDOSO, M.A.; CORRÊA, M.S.N.P. Humani zação do Atendimento Odontopediátrico: A Arte de uma Renovação. In: CORRÊA, Maria Salete Nahás Pires. Conduta clínica e psicológica na odontopediatria. 2. ed. São Paulo: Santos. 2013.

BRASIL. Ministério da Saúde. Secretaria de Atenção à Saúde. Departamento de Atenção Básica. Guia de recomendações para o uso de fluoretos no Brasil. Ministério da Saúde, Secretaria de Atenção à Saúde, Departamento de Atenção Básica. Brasília: Ministério da Saúde, 2009.

BRASIL. Ministério da Saúde. Secretaria de Atenção à Saúde. Departamento de Atenção Básica. Política Nacional de Atenção Básica. Ministério da Saúde, Secretaria de Atenção à Saúde, Departamento de Atenção Básica. 4. ed. Brasília: Ministério da Saúde, 2007. 68 p. Série E. Legislação de Saúde (Série Pactos pela Saúde 2006; v. 4). Disponível em:

http://bvsms.saude.gov.br/bvs/publicacoes.pacto.saude.v4.4e d.pdf. Acesso em: 11 jun. 2017.

BRASIL. Ministério da Saúde. Secretaria de Atenção à Saúde. Departamento de Atenção Básica. Projeto SB Brasil 2003: condições de saúde bucal da população brasileira 2002-2003: resultados principais. Ministério da Saúde, Secretaria de Atenção à Saúde, Departamento de Atenção Básica. Brasília: Ministério da Saúde, 2004.

BRASIL. Ministério da Saúde. Secretaria de Políticas de Saúde. Projeto Promoção da Saúde. As Cartas da Promoção de Saúde. Ministério da Saúde, Secretaria de Políticas de Saúde, Projeto Promoção da Saúde. Brasília: Ministério da Saúde, 2002.

BUENO, E.A.; MALHEIROS, R.T.; BALK, R.S. Promoção da saúde bucal: uma abordagem multidisciplinar. Rev Epidemiol Control Infect., v.2, n.3, p.115, 2012.

CORREA, A.L.; MACEDO, D.M.; ARAÚJO, M.V.A. Promoção em saúde Bucal na Creche Sorena. In: CONGRESSO DE EDUCAÇÃO EM SAÚDE DA AMAZÔNIA (COESA), 5., Belém, 2016. Anais... Belém: Universidade Federal do Pará, 2016. Disponível em:

http://www.coesa.ufpa.br/arquivos/2016/expandidos/extensao/ed ucacao_em_saude/EXT067.pdf. Acesso em: 05 mai. 2017.

FREIRE, M.C.M. Prevalência de cárie e fatores sócioeconômicos em pré-escolares: revisão da literatura. Rev bras odontol saúde coletiva, v.1, p.43-49, 2000.
HAUSEN, H.; SEPPA, L.; POUTANEN, R.; NIINIMAA, A.; LAHTI, S.; KÄRKKÄINEN, S.; PIETILÄ, I.

Noninvasive control of dental caries in children with active initial lesions. A randomized clinical trial. Caries Res., v.41, n.5, p. 384-391, 2007.

IGLESIAS, A.; DALBELLO-ARAÚJO, M. As concepções de promoção de saúde e suas implicações. Cad. Saúde Colet., v.19, n.3, p. 291-298, 2011.

MESQUINI, M.A.; MOLINARI, S.L.; PRADO, I.M.M. Educação em saúde bucal: uma proposta para abordagem no Ensino Fundamental e Médio. Arq Mudi., v.10, n.3, p.1622, 2006.

MOYSÉS, S.P.T.; WATT, R. Promoção de saúde bucal: definições. In: BUISCH, Y.P. Promoção de saúde bucal na clínica odontológica. São Paulo: Artes Médicas, 2000. p.3-17.

MURRAY, J. A saúde bucal no século XXI. In: MURRAY, J.J.; NUNN, J.H.; STEELE, J.G. Doenças orais: medidas preventivas. 4.ed. Rio de Janeiro: Guanabara Koogan, 2005. p.3-5.

NORMAN, A.H. Promoção da saúde: um desafio para a atenção primária. Rev Bras Med Fam Comunidade, v. 8, n.28, p. 153-154, 2013.

OLIVEIRA, B.H.; SANTOS, A.P.P.; NADANOVSKY, P. Uso de dentifrícios fluoretados por pré-escolares: o que os pediatras precisam saber? Residência Pediátrica, v.2, n.2, p.12-19, 2012.

OLIVEIRA, J.C.C. Atividades lúdicas na Odontopediatria: uma breve revisão da literatura. Rev. bras. odontol., Rio de Janeiro, v.71, n.1, p. 103-107, 2014.

SANTOS, K.T.; GARBIN, A.J.I.; GARBIN, C.A.S. Saúde bucal nas escolas: relato de experiência. Rev. Ciênc. Ext., v.8, n.1, p.161-169, 2012.

SÍCOLI, J. L.; NASCIMENTO, P. R. Promoção de saúde: concepções, princípios e operacionalização. Interface Comunic,Saúde, Educ., v.7, n.12, p.91-112, 2003.

Disponível em:

http://www.scielo.br/pdf/icse/v7n12/v7n12a07.pdf. Acesso em: 10 nov. 2018.

SIQUEIRA, M.F.G.; JARDIM, M.C.A.M.;

VASCONCELOS, L.C.S.; VASCONCELOS, L.C.

Evaluation of an oral health program for children in early childhood. Rev. odonto ciênc., v.25, n.4, p.350-354, 2010.

UNIVERSIDADE FEDERAL DO PARÁ. Instituto de Ciências da Saúde. Faculdade de Odontologia. Projeto Pedagógico do Curso de Odontologia. Belém. 2000. Disponível em:

http://ufpa.br/odontologia/index.php?option=com_content\& view=article\&id=2\&Itemid=4 Acesso em: 15 jun. 2017 .

VENÂNCIO, D.R.; GIBILINI, C.; BATISTA M.J.; GONÇALO C.S.; SOUSA, M.L.R. Promoção da saúde bucal: desenvolvendo material lúdico para crianças na faixa etária pré-escolar. J Health Sci Inst., v.29, n.3, p.153-6, 2011.

WORLD HEALTH ORGANIZATION. Regional Office for Europe. Health promotion: a discussion document on the concept and principles: summary report of the Working Group on Concept and Principles of Health Promotion, Copenhagen, 9-13 July 1984. Copenhagen: WHO Regional Office for Europe. Disponível em:

http://www.who.int/iris/handle/10665/107835. Acesso em: 20 mai. 2017. 